\title{
Long-term Outcomes and Prognostic Factors in Papillary Thyroid Microcarcinoma Patients with Distant Metastases
}

\section{Huai-Yu Weng}

Shanghai 6th Peoples Hospital Affiliated to Shanghai Jiaotong University School of Medicine

Ting Yan

Shanghai 6th Peoples Hospital Affiliated to Shanghai Jiaotong University School of Medicine

Wang-Wang Qiu

Shanghai 6th Peoples Hospital Affiliated to Shanghai Jiaotong University School of Medicine

Chuang Xi

Shanghai 6th Peoples Hospital Affiliated to Shanghai Jiaotong University School of Medicine

Li-Ying Hou

Shanghai 6th Peoples Hospital Affiliated to Shanghai Jiaotong University School of Medicine

Zhi-Li Yang

Shanghai 6th Peoples Hospital Affiliated to Shanghai Jiaotong University School of Medicine

Zhong-Ling Qiu ( $\nabla$ qiuzhongling123@163.com )

Shanghai 6th Peoples Hospital Affiliated to Shanghai Jiaotong University School of Medicine https://orcid.org/0000-0003-0834-5217

\section{Research Article}

Keywords: distant metastases, papillary thyroid microcarcinoma, radioiodine therapy, overall survival, progression-free survival

Posted Date: July 26th, 2021

DOI: https://doi.org/10.21203/rs.3.rs-734874/v1

License: (c) (i) This work is licensed under a Creative Commons Attribution 4.0 International License. Read Full License

Version of Record: A version of this preprint was published at Endocrine on October 26th, 2021. See the published version at https://doi.org/10.1007/s12020021-02906-8. 


\section{Abstract}

Purposes

Distant metastasis from papillary thyroid microcarcinoma (PTMC) is extremely rare and the long-term outcome and independent prognostic factors remain unclear.The present study aimed to investigate clinicopathological characteristics and evaluate the long-term outcomes and prognostic factors of PTMC patients with distant metastases (DM) who underwent surgery and radioactive iodine $\left({ }^{131} \mathrm{I}\right)$ treatment.

Methods

We retrospectively reviewed the medical records of 13,441 patients with thyroid cancer (including 1,697 cases with PTMC) who underwent ${ }^{131}$ I treatment at our institution between January 2008 and December 2019. PTMC patients with distant metastases with sufficient clinical follow-up data were enrolled in this cohort study. The overall survival (OS) and progression-free survival (PFS) were analyzed by the Kaplan-Meier method and the prognostic factors were assessed by Cox proportional hazards.

Results

Thirty-three PTMC patients with DM were enrolled in this study. The median follow-up was 75 months (range: 5-151 months). The 5-year and 10-year OS rates were $96.97 \%$ and $81.41 \%$, respectively, and the 5-year and 10 -year PFS rates were $90.46 \%$ and $69.68 \%$, respectively. Multivariate analysis showed that male $\operatorname{sex}(P=0.005)$, radioactive iodine refractory PTMC $(P=0.033)$ and symptomatic DM $(P=0.022)$ were significantly associated with worse 10-year PFS in PTMC patients with DM. No independent predictor related to poor 10-year OS found in the present study.

Conclusions

The prognosis of PTMC patients becomes worse after the development of DM. Male sex, radioactive iodine refractory PTMC and symptomatic DM were identified as independent factors associated with PFS.

\section{Introduction}

The incidence of thyroid carcinoma has increased sharply worldwide in recent decades(1). Papillary thyroid carcinoma (PTC) constitutes a major portion of thyroid cancer,takes up more than $80 \%$ of all thyroid malignancies $(2,3)$. Owing to the development of new diagnostic techniques with increased sensitivity, such as ultrasonography (US), fine-needle aspiration, computed tomography (CT), and magnetic resonance imaging scanning (MRI),over $50 \%$ of the new cases of thyroid cancers are papillary thyroid microcarcinoma (PTMC), which is defined by the World Health Organization (WHO) as a subtype of PTC measuring $\leq 1 \mathrm{~cm}$ in greatest dimension $(4,5)$. The treatment for patients with PTMC remains controversial because of the excellent prognosis of PTMC which has a 10 -year survival rate of over $90 \%$ and a recurrence rate of approximately $10 \%(2,6)$.Given the indolent behaviors and favorable outcomes of PTMC, an active surveillance approach has been recommended by the American Thyroid Association guidelines as an alternative option for patients with lowrisk PTMC(7).However, there are still a minority of high-risk PTMC cases with aggressive tumor features that are related to the increased risk of recurrence, such as extrathyroidal extension (ETE), lymph node metastases and distant metastases(8-10). Therefore, thyroid surgery is still the dominant treatment modility for PTMC

The prognosis of differentiated thyroid cancer (DTC) patients become much worse after the appearance of distant metastases. Therefore, even though distant metastases from PTMC are fairly rare with an incidence rate rangeing from $0-2.8 \%$ according to different investigations(9, 11-13), evaluating the long-term outcomes and prognostic factors of PTMC patients with distant metastases is important. Only a few case reports about PTMC cases with DM and several studies with a small number of patients have been published. Only one independent study evaluated the clinicopathological features and the risk factors for distant metastases in 12 patients with PTMC.This study revealed two independent risk factors for predicting distant metastasis of PTMC,including the presence of extranodal extension and the pathologic subtype of metastatic cervical lymph nodes(11). No independent study on the long-term outcomes and prognostic factors of PTMC patients with DM has been reported so far.

We performed the present retrospective study on 33 PTMC patients with DM who received radioactive iodine (RAI) treatment after thyroid surgery between 2008 and 2019 at our institution. We investigated clinical and histological characteristics of PTMC patients with DM and evaluate long-term outcomes and prognostic factors associated with overall survival (OS) and progression-free survival (PFS) after thyroidectomy and RAI therapy.

\section{Materials And Methods Patients selection}

This retrospective study was conducted in Shanghai Sixth People's Hospital Affiliated to Shanghai Jiao Tong University. From January 2008 to December 2019 , we reviewed the clinical follow-up data of a total of 13,441 patients who underwent total or near-total thyroidectomy followed by radioiodine therapy in our institution,including 1,697 PTMC patients. The inclusion criteria were as follows: (i) postoperative pathological diagnosis with PTMC;(ii) ${ }^{131}$ I treatment after total or near-total thyroidectomy; and (iii) diagnosis of distant metastases. Patients with a history of other malignancies or insufficient clinical follow-up data were excluded from the study. After excluding one patient with synchronous primary breast cancer and three patients with inadequate clinical follow-up data, 33 cases with distant metastatic PTMC were included in this study.

\section{Assessment of variables}


Age was recorded on the date of the initial diagnosis of distant metastases, and the cutoff age for risk stratification was set at 55 years as recommended by the Eighth Edition of the American Joint Committee on Cancer (14).The pathology of PTMC was divided into two subtypes: classical PTMC (C-PTMC) and follicular variant PTMC (FV-PTMC). The timing of the metastasis diagnosis was classified into two categories: distant metastases at initial presentation (the metastases were detected before or within 6 months after thyroid surgery) and delayed distant metastases (the metastases were confirmed more than 6 months after thyroidectomy) $(15,16)$.lodine avidity was determined by visual uptake in the known site of metastatic lesions by whole-body scanning $\left({ }^{131}\right.$ IWBS) and/or ${ }^{131} \mathrm{I}$-single-photon emission computed tomography/computed tomography $\left({ }^{131} \mathrm{I}\right.$-SPECT/CT) after radioiodine treatment. Non- ${ }^{131} \mathrm{I}$ avidity was defined as absence of visual uptake on ${ }^{131}$ I-WBS after ${ }^{131}$ I therapy or uptake in < $10 \%$ of multiple metastatic lesions on ${ }^{131}$ I-WBS $(17,18)$.

\section{Diagnostic criteria for PTMC patients with distant metastases}

As reported in our previous studies, the diagnosis of distant metastases had to fulfill at least one of the following three criteria: criterion I: distant metastases identified by serial cross-sectional imaging such as CT, MRI, and/or ${ }^{18} \mathrm{~F}-\mathrm{FDG}-\mathrm{PET} / \mathrm{CT}$, and the biopsies or postoperative pathological findings were compatible with distant metastases of PTMC origin; criterion II: distant metastatic ${ }^{131}$ I uptake on post radioiodine therapy ${ }^{131} \mathrm{I}$-WBS, in combination with at least one positive imaging result, including CT, MRI, and ${ }^{131} \mathrm{I}$-SPECT/CT; and criterion III: no ${ }^{131}$ I uptake by distant metastatic lesions on ${ }^{131} \mathrm{I}$-WBS, but positive results on ${ }^{18}$ F-FDG-PET/CT scans accompanied by elevated serum thyroglobulin ( $\mathrm{Tg}$ ) or progressively increased thyroglobulin antibody (TgAb) levels(19).

\section{Treatment protocol}

All PTMC patients underwent total or near-total thyroidectomy combined with neck lymph node dissection,followed by ${ }^{131}$ I therapy at $1-6$ months after surgery. Before ${ }^{131}$ I treatment, each patient was provided a low-iodine diet and began levothyroxine withdrawal for at least 2 weeks so that the level of thyroidstimulating hormone (TSH) increased to more than $30 \mathrm{mIU} / \mathrm{L}$. Medical examinations before RAl treatment included serial measurements of TSH, free triiodothyronine (FT3), free thyroxine (FT4), Tg, TgAb, neck ultrasonography, CT, and/or MRI scans were routinely performed. For patients with unknown distant metastases status before initial ${ }^{131}$ I treatment, the first oral dose of $3.7 \mathrm{GBq}(100 \mathrm{mCi})$ of ${ }^{131}$ I was administered for the remnant ablation and the subsequent oral doses of $5.55-7.40 \mathrm{GBq}(150-200 \mathrm{mCi})$ of ${ }^{131}$ I were administered to treat the distant metastatic lesions. For patients with distant metastatic disease confirmed before ablating residual thyroid tissue, a dose of $5.55-7.40 \mathrm{GBq}(150-200 \mathrm{mCi})$ of ${ }^{131}$ I was taken orally to ablate remnants and to treat distant metastatic foci. ${ }^{131} \mathrm{I}$-WBS and/or ${ }^{131} \mathrm{I}$-SPECT/CT fusion imaging was routinely performed $4-7$ days after therapeutic ${ }^{131} \mathrm{I}$ administration. The intervals for repeated ${ }^{131}$ I treatments varied from 4 to 12 months. In cases in which ${ }^{131} \mathrm{I}$-WBS showed non- ${ }^{131}$ I-avid distant metastases, the repeated ${ }^{131} \mathrm{I}$ treatment was suspended after complete residual thyroid tissue ablation.

\section{Diagnostic criteria for radioiodine refractory (RR-PTMC)}

According to the 2015 guidelines of the American Thyroid Association (7), patients with radioiodine refractory differentiated thyroid cancer can be classified into one of the following four categories: (i) the malignant/metastatic tissue does not concentrate radioiodine; (ii) the primary and/or metastatic lesions lose the ability to take up ${ }^{131}$ I after previous evidence of uptake; (iii) ${ }^{131}$ I concentration is present in some lesions but not in others; and (iv) primary/metastatic disease progresses within one year despite substantial uptake of ${ }^{131} \mathrm{I}$.

\section{Other adjuvant treatment modalities}

Targeted chemotherapy, such as tyrosine kinase inhibitors (TKIs), holds promise in the treatment of RR-PTMC patients with distant metastases, and sorafenib and lenvatinib are the only two to have been approved for treating radioiodine refractory differentiated thyroid cancer by the US Food and Drug Administration (FDA)(20). In our study, sorafenib was used to treat progressive RR-PTMC with distant metastases in two patients, and lenvatinib was used for treatment of RR-PTMC in one patient. Three patients had palliative surgery before RAl ablation, while palliative surgery was performed in two patients after ${ }^{131}$ I ablating thyroid remnants. In addition, four patients received external radiotherapy. Only one patient underwent interventional therapy.Similarly,chemotherapy was conducted in only one patient. Other therapies such as bisphosphonates were not applied for the treatment of PTMC patients with distant metastases in this study.

\section{Evaluation of distant metastatic progression}

As described in our previous studies(19),the effectiveness of radioactive iodine therapy was assessed according to the Response Evaluation Criteria in Solid Tumors Guideline Version 1.1 (RECIST v1.1) (21) for pulmonary metastases and the MDA criteria(22) for bone metastases, which was divided into two types: progressive disease(PD) and non-progressive disease(non-PD). For pulmonary metastasis, an absolute increase of at least $5 \mathrm{~mm}$ as well as a $20 \%$ increase in the sum of diameters for all measured target lesions is defined as PD. The appearance of new lesions was also considered as PD.For PD of PTMC patients with bone metastases, $a>25 \%$ increase and $>25 \%$ subjective increase were required for the size of measurable lesions and ill-defined lesions on CT or MRI,respectively. New bone metastases were also regarded as PD.

\section{Follow-up}

After ${ }^{131}$ I therapies, routine follow-up was carried out for every patient. Routine follow-up evaluation consisted of the measurement of FT3, FT4, TSH, Tg, and TgAb levels every 3-6 months, as well as neck US every 3-6 months. For patients with lung metastases, chest CT was performed every 6-12 months to evaluate the state of pulmonary metastatic foci. To assess the status of bone metastases and other extrapulmonary metastatic tissue, imaging examinations, including $\mathrm{CT}, \mathrm{MRI}$, or 18F-FDG-PET/CT, were conducted at least once per year. OS was defined as the time from the initial detection of distant metastatic lesions to death from any cause or end of follow-up. PFS was defined as the time from initial detection of distant metastases to progression from any cause or end of follow-up.

\section{Statistical analysis}


All statistical analyses were performed using SPSS 26.0 (SPSS software,IBM Corporation, Armonk, NY, USA), MedCalc 17.0 (MedCalc Software, Mariakerke, Belgium) and Prism 7.0 (GraphPad Software, San Diego, CA, USA). Quantitative variables were expressed as means \pm standard deviation (SD) with range and median,while qualitative variables were presented as exact numbers and percentages. The Kaplan-Meier method was applied to analyze OS and PFS survival curves. Univariate analyses were conducted for evaluating survival outcomes and prognostic factors for OS rate and PFS rate, and the differences between cohorts were analyzed for significance by the log-rank test. To identify independent factors associated with OS and PFS, parameters with a P value $<0.1$ in univariate analyses were selected for multivariate analyses, which were computed by the Cox proportional hazards model. A P value $<0.05$ were considered to be statistically significant.

\section{Results}

\section{Patient characteristics}

Among the 1,697 patients with PTMC, 33 were confirmed with a diagnosis of distant metastases. The incidence of PTMC patients with distant metastases was $1.94 \%$. The clinicopathological characteristics of the 33 PTMC patients with DM are listed in Table 1. All patients enrolled in this study showed welldifferentiated PTMC.

\section{Diagnoses and characteristics of distant metastases}

Among the 33 PTMC patients with DM, 7 patients were diagnosed according to Criterion I,23 cases were diagnosed based on Criterion II and 3 patients were confirmed by Criterion III. In addition, 28 (84.85\%) patients presented with asymptomatic DM, and only 5(15.15\%) cases showed clinical symptoms, including 1 patient with difficulty breathing, 1 patient with dry cough, 1 patient with bone pain, 1 patient with pathologic fractures, and 1 patient with bone pain and spinal cord compressive simultaneously. Regarding the sites of distant metastases, 21 (63.64\%) cases showed only pulmonary metastases, 8 (24.24\%) patients showed bone metastases alone and 2 (6.06\%) patients presented with multiple organ metastases, including 1 with synchronous lung and bone metastases and 1 with synchronous lung, brain and liver metastases. Metastases in other single sites occurred in two (6.06\%) patients, including one with brain metastasis and one with right forearm soft tissue metastasis(Table1).

\section{Changes in serum suppressed $\mathrm{Tg}(\mathrm{s}-\mathrm{Tg})$ levels}

Among the 33 PTMC patients with distant metastases, only 7 (21.21\%) cases presented PD based on anatomical imaging changes, while $26(78.79)$ patients showed absence of PD. During follow-up, TSH was suppressed to less than $0.1 \mathrm{mlU} / \mathrm{mL}$ in all patients. We compared the s-Tg levels measured six months after RAl ablation and the final s-Tg measurement at follow-up end. Among the total 33 patients, 31 cases presented positive s-Tg and 2 patients showed undetectable s-Tg, including one with increased $\mathrm{TgAb}$ and one with persistently high $\mathrm{TgAb}(>4000 \mathrm{IU} / \mathrm{mL}$ ). Among the 31 (93.94\%) cases with positive s-Tg, the mean s-Tg changed from 52.39 (range: $0.36-455.00) \mathrm{ng} / \mathrm{mL}$ to 388.57 (range: $0.24-5002.00) \mathrm{ng} / \mathrm{mL}$. Of these 31 cases, 6 (19.4\%) cases were from the PD group and the other 25 (80.6\%) were from the non-PD group, which was assessed based on anatomical imaging. The changes of s-Tg levels in the PD group and non-PD group are presented in Figure 1A and 1B, respectively. In the PD group, the levels of s-Tg showed an increase in four (57.14\%) patients and a decrease in two (28.57\%) patients,and one (14.29\%) patient showed undetectable s-Tg due to the persistently high $\mathrm{TgAb}$ level. The mean s-Tg in six patients whose s-Tg levels were detectable changed from 100.42 (range: $6.58-255.20$ ) ng/mL to 1943.35 (range: $3.84-5002.00$ ) $\mathrm{ng} / \mathrm{mL} . \mathrm{In}$ the non-PD group, 23 (88.46\%) cases presented decreased s-Tg and only 2 (7.69\%) had increased s-Tg, and 1 (3.85\%) case presented undetectable s-Tg because of increased TgAb level. The average s-Tg level in 25 patients with detectable s-Tg decreased from 40.86 (range: $0.36-455.00) \mathrm{ng} / \mathrm{mL}$ to $15.43(\mathrm{range:} 0.24-158.00) \mathrm{ng} / \mathrm{mL}$.

\section{Response to RA/ treatment}

As shown in Table 1, the mean number of RAl courses was 2.48 \pm 1.70 (range: 1-9 times) and the mean cumulative RAl dose was $15.34 \pm 12.82 \mathrm{GBq}$ (range: $3.70-64.75 \mathrm{GBq})$. After the 33 patients received an oral therapeutic dose of ${ }^{131} \mathrm{I}$, ${ }^{131}$ I avidity was shown on ${ }^{131}$ I-WBS in 25 (75.76\%) patients and non-131 I avidity was presented in 8 (24.24\%) patients. Based on the evaluation criteria for RR-PTMC, 15 (45.45\%) cases were categorized as RR-PTMC and 18 (54.55\%) were regarded as non-RR-PTMC. Among the 15 RR-PTMC patients, 6 patients were confirmed by criterion (i), four cases were diagnosed according to criterion (ii),three patients were determined based on criterion (iii), and only two cases were confirmed on the basis of criterion (iv).

\section{Survival analysis for PTMC patients with distant metastases}

The mean duration of follow-up was $74.67 \pm 36.65$ months (range from 5 to 151 months) from the initial diagnosis of distant metastases. Over the course of follow-up,4 (12.12\%) patients died and 29 (87.88\%) survived. Of the four cases,one patient died at 73 months after the diagnosis of lung metastasis,two patients died at 5 and 84 months after the confirmation of bone metastases, and one patient died 62 months after the diagnosis of synchronous lung and bone metastases. According to the RECIST v1.1 criteria for pulmonary metastases and the MDA criteria for bone metastases,three patients showed PD at the end of the 5-year follow-up and seven cases presented PD at the end of the 10-year follow-up. The 5- and 10-year OS rates were $96.97 \%$ and $81.41 \%$, respectively (Figure 2A). The 5 - and 10 -year PFS rates were $90.46 \%$ and $69.68 \%$, respectively (Figure 2B).

\section{Prognostic factors for PTMC patients with distant metastases}

The results of the univariate and multivariate analyses of 10-year OS rates and 10-year PFS rates are displayed in Table 2-4. In univariate analysis, age at initial diagnosis of distant metastases ( $\mathrm{P} \otimes 0.001)$ and RR-PTMC status $(\mathrm{P}=0.011)$ were significantly associated with the 10 -year OS rates. Patients older than 55 years old and with RR-PTMC had lower 10-year OS rates compared with those younger than 55 years old and with non-RR-PTMC, respectively (Figure 3). However, age $(P=0.006)$, sex $(P=0.004)$, RR-PTMC status $(P=0.006)$, adjuvant treatment modalities ( $\mathrm{P} \otimes 0.001)$ and symptoms of distant metastases $(P \otimes 0.001)$ were significantly related to the 10-year PFS rates (Figure 4). Patients over 55 years old, male patients and patients with RR-PTMC, RAI plus other adjuvant 
therapies and symptomatic distant metastases had worse 10-year PFS rates than those younger 55 years of age, female patients, and patients with non-RRPTMC, RAl alone and asymptomatic distant metastases, respectively. Among the selected factors $(p<0.1)$ from univariate analysis, male sex $(P=0.005)$, RRPTMC $(P=0.033)$ and symptomatic distant metastases $(P=0.022)$ were statistically significant poor prognostic factors for 10 -year $P F S$ rates by Cox regression analysis (Table 4); the rest were not significantly associated with 10-year PFS rates. No independent prognostic factor was found for the 10-year OS rates.

\section{Discussion}

Few reports have examined the incidence and clinicopathological characteristics of PTMC patients with distant metastases,in part because of the low incidence of these cases. Most published studies have been either individual case reports or small case series, and no study has reported on the long-term outcomes and prognostic factors of PTMC cases with DM. In this study, we investigated the clinicopathological features of 33 patients with distant metastases from PTMC, after surgery and radioiodine treatment. This study is the first to focus on the long-term outcomes and prognostic factors of PTMC patients with DM. The incidence rate of distant organ metastases from PTMC in this study was $1.94 \%$, which was in accordance with previous reports showing that distant metastases occurred in $0-2.8 \%$ of PTMC patients $(9,11-13)$. One important distinction between our present study and previous reports is that we selected candidates from PTMC patients who had undergone RAI therapy after thyroidectomy.

PTC is a disease with a generally good outcome, but PTC patients presenting with distant metastases show less favorable outcomes. A study conducted in Japan by Sugitani et al. enrolled 86 PTC patients with distant metastases and found that the 5-year and 10-year survival rates were $65 \%$ and $45 \%$,respectively(23). Similarly, PTMC was generally associated with an excellent prognosis, with 10 -year overall survival rate of an approximately $95 \%$,while Yu et al. reported that PTMC patients with distant metastases had worse prognosis with a 10-year survival of $68 \%$ (2). In the current study, the 5-and 10 -year OS rates for PTMC patients with distant metastases were $96.97 \%$ and $81.41 \%$, respectively. Compared with PTC patients with distant metastases, PTMC patients with distant metastases have a better prognosis $(2,23)$. In addition, there was discrepancy in the survival rate of PTMC patients with distant metastases between other studies and this study. The 10-year OS rate for PTMC patients with distant metastases in our investigation was approximately $13 \%$ higher than the result in previous study conducted by Yu et al.(2). This discrepancy may be explained by the fact that all patients in our cohort had undergone

${ }^{131}$ I treatment after thyroid surgery and most of them (75.76\%) showed ${ }^{131}$ I -avidity. In this study, the 5 -year PFS was $90.46 \%$ and the 10 -year PFS was $69.68 \%$, which also demonstrated the poor prognosis caused by distant metastatic disease of PTMC origin.

The prevalence of PTC is much higher in female patients than in male patients(24-28). Consistent with these studies, in our patient group,the occurrence of PTMC with distant metastases among females was 2.67 times higher than males (24:9). However, the reasons underlying these differences between sexes are unknown. Whether sex is an independent factor related to the prognosis of PTC remains unclear. In some studies, male sex was regarded as a worse prognostic factor for PTC than female sex $(25,26)$. Nevertheless, other studies found male sex was not a significant prognostic factor for survival in PTC patients $(27,28)$. A few studies even reported conflicting results. Lee et al. analyzed 2,930 cases of PTC and concluded that male sex was an independent poor prognostic factor in PTC $1 \mathrm{~cm}$, yet it was not an independent prognostic factor in PTMC(24). In the present study, we determined that male sex was a statistically significant poor prognostic factor for 10-year PFS rate in both the univariate and multivariate analyses. Therefore, male PTMC patients with DM need close surveillance and aggressive treatment.

Radioiodine refractory differentiated thyroid carcinoma (RR-DTC) represents a major therapeutic challenge in thyroid cancer medicine, and is mainly caused by the BRAF ${ }^{\mathrm{V} 600 \mathrm{E}}$ mutation that leads to the abnormal activation of the MAPK pathway $(29,30)$. Although RR-DTC occurs in less than $5 \%$ of patients with DTC, it is more commonly found in patients with distant metastatic disease, of whom approximately $60 \%$ are diagnosed with RR-DTC(19, 30). However, 15 (45.45\%) PTMC patients with distant metastases were confirmed to be refractory to radioiodine in the present study, which was approximately $15 \%$ lower than that in studies mentioned above. This suggested that PTMC patients with distant metastases had lower levels of dedifferentiation than DTC cases with distant metastases. Our previous study had demonstrated that elderly RR-DTC patients ( $\geq 65$ years old) with distant metastases had poorer prognosis than elderly non-RR-DTC patients and revealed that RR-DTC was an independent prognostic factor associated with OS(19).In contrast, in this study, we found RR-PTMC was not an independent factor for OS. These contradictory results might be from differences in populations with different ages, pathological types and tumor sizes. However, we also confirmed that RR-PTMC patients with distant metastases had a poorer prognosis and showed that RR-PTMC was a predictive factor for 10-year PFS indicating that RR-PTMC could still influence the prognosis of PTMC cases with distant metastases.

In our previous study,nearly half of the elderly DTC patients with distant metastases were symptomatic at initial diagnosis(19). Kim et al. analyzed 127 thyroid carcinoma patients with initial distant metastasis in Korea and found that clinical systemic symptoms were present in approximately $10 \%$ of patients(31). In this study, we found that $5(15.15 \%)$ patients had symptoms of distant metastasis, while most patients (84.85\%) were asymptomatic at initial diagnosis. One possible explanation for this finding is the development of screening methods. Kim et al. revealed that thyroid carcinoma patients with symptomatic initial distant metastases had a higher disease-specific death rate compared with that of the symptomatic groups after 2004(31). Even though there was no significant difference in the 10-year rates between the symptomatic group and the asymptomatic group in our study, statistically significant difference was present in the 10-year PFS rates between these two groups. Thus, early detection by screening was necessary and helpful in PTMC patients with DM.

Our study has several limitations. First, the main limitation of the study is its retrospective design, and future prospective studies could address this issue. Second, because of the long study period, some clinical data were missing, which might have caused bias in data selection. Third, because this study was a single-center investigation, further research is needed to confirm the reproducibility of our findings in other patient cohorts, including those of other races. In addition, because of the limited number of patients,the longest follow-up times of some subgroups in this study were less than 10 years,which might cause bias when comparing the survival rates of different subgroups. Therefore, the follow-up time and sample size need to be increased in future studies.

\section{Conclusion}


To the best of our knowledge, this is first study to assess the long-term outcomes and prognostic factors of distant metastases in PTMC patients who underwent surgery and RAI therapy. We found that the prognosis of PTMC became worse after the development of distant metastases,with 5- and 10-year OS rates of $96.97 \%$ and $81.41 \%$,respectively, and 5 - and 10 -year PFS rates of $90.46 \%$ and $69.68 \%$,respectively. Furthermore,we confirmed that male sex, presence of RR-PTMC and symptomatic distant metastases were predictive factors for worse 10-year PFS,yet these were not independent factors related to worse 10year OS. Hence, closer surveillance and more aggressive treatment are warranted for male patients, symptomatic patients and RR-PTMC patients with DM. Further prospective trials are needed to confirm our findings and the sample size should be increased.

\section{Declarations}

\section{Acknowledgment}

The authors thank Gabrielle White Wolf, PhD, from Liwen Bianji (Edanz) (www.liwenbianji.cn/), for editing the English text of a draft of this manuscript.

\section{Funding}

This work was supported by the National Natural Science Foundation of China (grant

number 81771865), the Natural Science Foundation of Shanghai (grant number 21ZR1448500) and the Project from Shanghai Sixth People's Hospital (grant number YNLC201905)

\section{Authors contribution}

H.Y.W, Z.L.Q. and Z.L.Y designed the study. H.Y.W,T.Y and W.W.Q conducted the statistical analysis. H.Y.W,C.X and L.Y.H collected the clinical data. H.Y.W wrote the whole paper. H.Y.W, Z.L.Q and Z.L.Y supervised and edited the paper. All authors read and approved the final paper.

\section{Conflict of interest statement}

The authors declare that they have no conflict of interest.

\section{Data availability statement}

The data sets used and/or analysed in the present study are available from the corresponding authors upon request.

\section{Ethical approval}

The study protocol was approved by the Ethics Committee of the Shanghai Jiao Tong

University Affiliated Sixth People's Hospital, Shanghai 200233, China.

\section{References}

1. Davies L, Welch HG. Increasing incidence of thyroid cancer in the United States, 1973-2002. Jama 295:2164-2167(2006).

https://doi.org/10.1001/jama.295.18.2164

2. Yu XM, Wan Y, Sippel RS, Chen H. Should all papillary thyroid microcarcinomas be aggressively treated? An analysis of 18,445 cases. Annals of surgery 254:653-660(2011). https://doi.org/10.1097/SLA.0b013e318230036d

3. Lim H, Devesa SS, Sosa JA, Check D, Kitahara CM. Trends in Thyroid Cancer Incidence and Mortality in the United States, 1974-2013. Jama 317:13381348(2017). https://doi.org/10.1001/jama.2017.2719

4. Gao M, Ge M, Ji Q, et al. 2016 Chinese expert consensus and guidelines for the diagnosis and treatment of papillary thyroid microcarcinoma. Cancer biology \& medicine 14:203-211(2017). https://doi.org/10.20892/j.issn.2095-3941.2017.0051

5. Chen AY, Jemal A, Ward EM. Increasing incidence of differentiated thyroid cancer in the United States, 1988-2005. Cancer 115:3801-3807(2009). https://doi.org/10.1002/cncr.24416

6. Giordano D, Gradoni P, Oretti G, Molina E, Ferri T. Treatment and prognostic factors of papillary thyroid microcarcinoma. Clinical otolaryngology : official journal of ENT-UK; official journal of Netherlands Society for Oto-Rhino-Laryngology \& Cervico-Facial Surgery 35:118-124(2010). https://doi.org/10.1111/j.1749-4486.2010.02085.x

7. Haugen BR, Alexander EK, Bible KC, et al. 2015 American Thyroid Association Management Guidelines for Adult Patients with Thyroid Nodules and Differentiated Thyroid Cancer: The American Thyroid Association Guidelines Task Force on Thyroid Nodules and Differentiated Thyroid Cancer. Thyroid : official journal of the American Thyroid Association 26:1-133(2016). https://doi.org/10.1089/thy.2015.0020

8. Pedrazzini L, Baroli A, Marzoli L, Guglielmi R, Papini E. Cancer recurrence in papillary thyroid microcarcinoma: a multivariate analysis on 231 patients with a 12-year follow-up. Minerva endocrinologica 38:269-279(2013). https://doi.org/10.1038/nutd.2013.31 
9. Zheng X, Wei S, Han Y, et al. Papillary microcarcinoma of the thyroid: clinical characteristics and BRAF(V600E) mutational status of 977 cases. Annals of surgical oncology 20:2266-2273(2013). https://doi.org/10.1245/s10434-012-2851-z

10. Chow SM, Law SC, Chan JK, Au SK, Yau S, Lau WH. Papillary microcarcinoma of the thyroid-Prognostic significance of lymph node metastasis and multifocality. Cancer 98:31-40(2003). https://doi.org/10.1002/cncr.11442

11. Jeon MJ, Kim WG, Choi YM, et al. Features Predictive of Distant Metastasis in Papillary Thyroid Microcarcinomas. Thyroid : official journal of the American Thyroid Association 26:161-168(2016). https://doi.org/10.1089/thy.2015.0375

12. Mazzaferri EL. Management of low-risk differentiated thyroid cancer. Endocrine practice : official journal of the American College of Endocrinology and the American Association of Clinical Endocrinologists 13:498-512(2007). https://doi.org/10.4158/ep.13.5.498

13. Ito Y, Miyauchi A, Kihara M, Higashiyama T, Kobayashi K, Miya A. Patient age is significantly related to the progression of papillary microcarcinoma of the thyroid under observation. Thyroid : official journal of the American Thyroid Association 24:27-34(2014). https://doi.org/10.1089/thy.2013.0367

14. Amin MB, Greene FL, Edge SB, et al. The Eighth Edition AJCC Cancer Staging Manual: Continuing to build a bridge from a population-based to a more "personalized" approach to cancer staging. CA: a cancer journal for clinicians 67:93-99(2017). https://doi.org/10.3322/caac.21388

15. Zhang XY, Song HJ, Qiu ZL, et al. Pulmonary metastases in children and adolescents with papillary thyroid cancer in China: prognostic factors and outcomes from treatment with (131)I. Endocrine 62:149-158(2018). https://doi.org/10.1007/s12020-018-1678-1

16. Song HJ, Qiu ZL, Shen CT, Wei WJ, Luo QY. Pulmonary metastases in differentiated thyroid cancer: efficacy of radioiodine therapy and prognostic factors. European journal of endocrinology 173:399-408(2015). https://doi.org/10.1530/eje-15-0296

17. Choi YM, Kim WG, Kwon H, et al. Early prognostic factors at the time of diagnosis of bone metastasis in patients with bone metastases of differentiated thyroid carcinoma. European journal of endocrinology 175:165-172(2016). https://doi.org/10.1530/eje-16-0237

18. Sohn SY, Kim HI, Kim YN, Kim TH, Kim SW, Chung JH. Prognostic indicators of outcomes in patients with lung metastases from differentiated thyroid carcinoma during long-term follow-up. Clin Endocrinol (Oxf) 88:318-326(2018). https://doi.org/10.1111/cen.13489

19. Qiu ZL, Shen CT, Sun ZK, et al. Long-Term Outcomes and Prognoses of Elderly Patients ( $\geq 65$-Years-Old) With Distant Metastases From WellDifferentiated Thyroid Cancer During Radioiodine Therapy and Follow-Up. Frontiers in endocrinology 11:588024(2020).

https://doi.org/10.3389/fendo.2020.588024

20. Wilson L, Huang W, Chen L, Ting J, Cao V. Cost Effectiveness of Lenvatinib, Sorafenib and Placebo in Treatment of Radioiodine-Refractory Differentiated Thyroid Cancer. Thyroid : official journal of the American Thyroid Association 27:1043-1052(2017). https://doi.org/10.1089/thy.2016.0572

21. Nishino M, Jagannathan JP, Ramaiya NH, Van den Abbeele AD. Revised RECIST guideline version 1.1: What oncologists want to know and what radiologists need to know. AJR American journal of roentgenology 195:281-289(2010). https://doi.org/10.2214/ajr.09.4110

22. Costelloe CM, Chuang HH, Madewell JE, Ueno NT. Cancer Response Criteria and Bone Metastases: RECIST 1.1, MDA and PERCIST. Journal of Cancer 1:80-92(2010). https://doi.org/10.7150/jca.1.80

23. Sugitani I, Fujimoto Y, Yamamoto N. Papillary thyroid carcinoma with distant metastases: survival predictors and the importance of local control. Surgery 143:35-42(2008). https://doi.org/10.1016/j.surg.2007.06.011

24. Lee YH, Lee YM, Sung TY, et al. Is Male Gender a Prognostic Factor for Papillary Thyroid Microcarcinoma? Annals of surgical oncology 24:19581964(2017). https://doi.org/10.1245/s10434-017-5788-4

25. Lin JD, Hsueh C, Chao TC. Long-Term Follow-Up of the Therapeutic Outcomes for Papillary Thyroid Carcinoma With Distant Metastasis. Medicine (Baltimore) 94:e1063(2015). https://doi.org/10.1097/md.0000000000001063

26. Ding J, Wu W, Fang J, Zhao J, Jiang L. Male sex is associated with aggressive behaviour and poor prognosis in Chinese papillary thyroid carcinoma. Scientific reports 10:4141(2020). https://doi.org/10.1038/s41598-020-60199-9

27. Toniato A, Boschin I, Casara D, Mazzarotto R, Rubello D, Pelizzo M. Papillary thyroid carcinoma: factors influencing recurrence and survival. Annals of surgical oncology 15:1518-1522(2008). https://doi.org/10.1245/s10434-008-9859-4

28. Grogan $\mathrm{RH}$, Kaplan SP, Cao H, et al. A study of recurrence and death from papillary thyroid cancer with 27 years of median follow-up. Surgery 154:14361446; discussion 1446-1437(2013). https://doi.org/10.1016/j.surg.2013.07.008

29. Xing M. Molecular pathogenesis and mechanisms of thyroid cancer. Nature reviews Cancer 13:184-199(2013). https://doi.org/10.1038/nrc3431

30. Jin Y, Van Nostrand D, Cheng L, Liu M, Chen L. Radioiodine refractory differentiated thyroid cancer. Critical reviews in oncology/hematology 125:111120(2018). https://doi.org/10.1016/j.critrevonc.2018.03.012 
31. Kim H, Park SY, Jung J, et al. Improved survival after early detection of asymptomatic distant metastasis in patients with thyroid cancer. Scientific reports 9:18745(2019). https://doi.org/10.1038/s41598-019-55370-w

\section{Tables}

Table 1. Clinicopatholodical characteristics of 33 PTMC patients with distant metastases 
Variables

$\mathrm{N}$ [\%

Age at diagnosis of distant metastases (years)(Mean $\pm S D \square M e d i a n \square R a n g e)$

$凶 55$

$\geq 55$

Sex

Male

Female

Number of thyroid surgeries

1

冈1

Pathological subtype

C-PTMC

FV-PTMC

Bilaterality

Absent

Present

ETE

No

Minimal/Gross

$11 \rrbracket 33.33 \rrbracket$

\section{$\mathrm{N}$ stage}

NO

N1a

N1b

Site of metastases

Only lung

21区63.64】

Only bone

$8 \otimes 24.24 】$

Only brain

Only soft tissue

Multiple sites

Time of distant metastasis

At initial presentation

23邓69.70区

Delayed distant metastases

10囚30.30区

Symptom of distant metastases at initial diagnosis

Asymptomatic

Symptomatic

Adjuvant treatment modalities of distant metastases

\begin{tabular}{lc} 
RAl treatment & $33(100)$ \\
\hline Palliative surgical treatment & $5(15.15)$ \\
\hline External radiotherapy & $4(12.12)$ \\
\hline Bone interventional therapy & $1(3.03)$ \\
Chemotherapy & $1(3.03)$ \\
TKIs therapy & $3(9.09)$
\end{tabular}

${ }^{131}$ I avidity

Yes

Page 9/17 


\begin{tabular}{|c|c|}
\hline No & $8 \rrbracket 24.24 \rrbracket$ \\
\hline \multicolumn{2}{|l|}{ RR-PTMC } \\
\hline Yes & $15 \llbracket 45.45 \rrbracket$ \\
\hline No & $18 \llbracket 54.55 \rrbracket$ \\
\hline Preablation stimulated $\mathrm{Tg}(\mathrm{ng} / \mathrm{mL})($ Mean $\pm S D \square M e d i a n \square R a n g e)$ & $1276.11 \pm 3346.99 \otimes 116.9,0.1-17934$ \\
\hline Number of courses for ${ }^{131}$ I therapy(Mean $\pm S D \square M e d i a n[R a n g e)$ & $2.48 \pm 1.70 \otimes 2 \otimes 1-9$ \\
\hline$\leq 3$ & $27 \rrbracket 81.82 \rrbracket$ \\
\hline$\llbracket 3$ & $6 \rrbracket 18.18 \rrbracket$ \\
\hline Cumulative dose of ${ }^{131}$ I activities(GBq)(Mean $\pm S D \square$ Median $\square$ Range) & $15.34 \pm 12.82,12.95,3.70-64.75$ \\
\hline$\leq 22.2(600 \mathrm{mCi})$ & $28 \llbracket 84.85 \rrbracket$ \\
\hline$\otimes 22.2(600 \mathrm{mCi})$ & $5 \bigotimes 15.15 \rrbracket$ \\
\hline \multicolumn{2}{|l|}{ Progression } \\
\hline No & $26 \bigotimes 78.79 \rrbracket$ \\
\hline Yes & $7 \llbracket 21.21 \rrbracket$ \\
\hline \multicolumn{2}{|l|}{ Death } \\
\hline No & $29 \llbracket 87.88 \rrbracket$ \\
\hline Yes & $4 \llbracket 12.12 \rrbracket$ \\
\hline Follow-up time(months)(Mean $\pm S D \square M e d i a n \square R a n g e)$ & $74.67 \pm 36.65 \rrbracket 75 \rrbracket 5-151$ \\
\hline
\end{tabular}

C-PTMC: classical papillary thyroid microcarcinoma; FV-PTMC: follicular variant papillary thyroid microcarcinoma; ETE: extrathyroidal extension; RR-PTMC: radioiodine refractory papillary thyroid microcarcinoma.

Table 2. Prognostic factors of 10-year OS rate for patients with PTMC and distant metastases in uivariate analysis 


\begin{tabular}{|c|c|c|c|c|c|c|c|}
\hline Variables & Patients & Deaths(\%) & $\begin{array}{l}\text { 10-year survival } \\
\text { rate(\%) }\end{array}$ & $\begin{array}{l}\text { Log- } \\
\text { rank }\end{array}$ & HR & $95 \% \mathrm{Cl}$ & $P$ value \\
\hline Age (years) & & & & 10.93 & & & $\llbracket 0.001$ \\
\hline$凶 55$ & 25 & $0 \otimes 0 \bigotimes$ & 100.00 & & - & - & \\
\hline$\geq 55$ & 8 & $4 \llbracket 50.0 \rrbracket$ & 43.75 & & - & - & \\
\hline Sex & & & & 3.36 & & & 0.067 \\
\hline Male & 9 & $2 \rrbracket 22.2 \rrbracket$ & 59.26 & & 1 & & \\
\hline Female & 24 & $2 \varangle 8.3 \rrbracket$ & 86.54 & & 0.08 & 0.006-1.192 & \\
\hline Number of thyroid surgeries & & & & 0.61 & & & 0.437 \\
\hline 1 & 27 & $4 \otimes 14.8 \rrbracket$ & 78.95 & & - & - & \\
\hline$\bigotimes 1$ & 6 & $0 \otimes 0 \bigotimes$ & 100.00 & & - & - & \\
\hline Pathological subtype & & & & 0.45 & & & 0.503 \\
\hline C-PTMC & 31 & $4 \llbracket 12.9 \rrbracket$ & 79.34 & & - & - & \\
\hline FV-PTMC & 2 & $0 \otimes 0 \bigotimes$ & 100.00 & & - & - & \\
\hline Bilaterality & & & & 2.04 & & & 0.153 \\
\hline absent & 20 & $4(20.0)$ & 73.15 & & - & - & \\
\hline present & 13 & $0(0)$ & 100.00 & & - & - & \\
\hline ETE & & & & $\nabla 0.01$ & & & 0.945 \\
\hline No & 22 & $3(13.6)$ & 83.10 & & 1 & & \\
\hline Minimal/Gross & 11 & $1(9.1)$ & 75.00 & & 0.92 & 0.099-8.607 & \\
\hline N stage & & & & 0.51 & & & 0.776 \\
\hline No & 10 & $2(20.0)$ & 72.92 & & 1 & & \\
\hline $\mathrm{N} 1 \mathrm{a}$ & 7 & $1(14.3)$ & 80.00 & & 0.76 & $0.056-10.461$ & \\
\hline $\mathrm{N} 1 \mathrm{~b}$ & 16 & $1(6.2)$ & 93.75 & & 0.43 & $0.045-4.036$ & \\
\hline Site of metastases & & & & 1.73 & & & 0.189 \\
\hline Pulmonary only & 21 & $1(4.8)$ & 90.00 & & 1 & & \\
\hline Extrapulmonary & 12 & $3(25.0)$ & 69.84 & & 3.80 & $0.520-27.711$ & \\
\hline Time of distant metastasis & & & & 0.06 & & & 0.803 \\
\hline Initial & 22 & $3(13.6)$ & 82.24 & & 1 & & \\
\hline Delayed & 11 & $1(9.1)$ & 80.00 & & 0.76 & $0.091-6.398$ & \\
\hline Symptom of distant metastases at initial diagnosis & & & & 3.26 & & & 0.071 \\
\hline Asymptomatic & 28 & $2(7.1)$ & 88.15 & & 1 & & \\
\hline Symptomatic & 5 & $2(40.0)$ & 53.33 & & 11.41 & $0.812-160.460$ & \\
\hline $\begin{array}{l}\text { Adjuvant treatment modalities of distant } \\
\text { metastases }\end{array}$ & & & & 3.84 & & & 0.050 \\
\hline RAl treatment & 24 & $1(4.2)$ & 90.91 & & 1 & & \\
\hline RAl combined with other treatment & 9 & $3(33.3)$ & 63.49 & & 8.50 & $0.999-72.276$ & \\
\hline${ }^{131}$ I avidity & & & & 1.95 & & & 0.162 \\
\hline No & 8 & $2(25.0)$ & 65.63 & & 1 & & \\
\hline Yes & 25 & $2(8.0)$ & 86.27 & & 0.18 & $0.017-1.986$ & \\
\hline RR-PTMC & & & & 6.41 & & & 0.011 \\
\hline No & 18 & $0(0)$ & 100.00 & & - & & \\
\hline Yes & 15 & $4(26.7)$ & 55.31 & & - & - & \\
\hline Number of courses for ${ }^{131}$ I therapy & & & & 0.11 & & & 0.737 \\
\hline$\leq 3$ & 27 & $3(11.1)$ & 82.39 & & 1 & & \\
\hline & & Page 11 & & & & & \\
\hline
\end{tabular}




\begin{tabular}{|c|c|c|c|c|c|c|}
\hline$\bigotimes 3$ & 6 & $1(16.7)$ & 75.00 & & 1.54 & $0.123-19.310$ \\
\hline Cumulative dose of ${ }^{131}$ I activivities(GBq) & & & & 0.15 & & 0.703 \\
\hline$\leq 22.2(600 \mathrm{mCi})$ & 28 & $3(10.7)$ & 82.50 & & 1 & \\
\hline$\otimes 22.2(600 \mathrm{mCi})$ & 5 & $1(20.0)$ & 75.00 & & 1.65 & $0.126-21.561$ \\
\hline
\end{tabular}

Table 3. Prognostic factors of 10-year PFS rate for patients with PTMC and distant metastases in uivariate analysis 


\begin{tabular}{|c|c|c|c|c|c|c|c|}
\hline Variables & Patients & $\mathrm{PD}(\%)$ & 10-year PFS rate(\%) & Log-rank & HR & $95 \% \mathrm{Cl}$ & $P$ value \\
\hline Age (years) & & & & 7.60 & & & 0.006 \\
\hline$\otimes 55$ & 25 & $2 \varangle 8.0 \rrbracket$ & 91.20 & & 1 & & \\
\hline$\geq 55$ & 8 & $5 \llbracket 62.5 \rrbracket$ & 29.17 & & 10.85 & $1.992-59.078$ & \\
\hline Sex & & & & 8.30 & & & 0.004 \\
\hline Male & 9 & $4 \llbracket 44.4 \rrbracket$ & 25.93 & & 1 & & \\
\hline Female & 24 & $3 \rrbracket 12.5 \rrbracket$ & 81.67 & & 0.05 & $0.007-0.390$ & \\
\hline Number of thyroid surgeries & & & & 1.21 & & & 0.272 \\
\hline 1 & 27 & $7 \rrbracket 25.9 \bigotimes$ & 65.51 & & - & & \\
\hline$\nabla 1$ & 6 & $0 \otimes 0 \bigotimes$ & 100.00 & & - & - & \\
\hline Pathological subtype & & & & 0.72 & & & 0.396 \\
\hline Classical & 31 & 6囚19.4囚 & 70.72 & & 1 & & \\
\hline Follicular variant & 2 & $1 \otimes 50.0 \bigotimes$ & 50.00 & & 3.71 & $0.180-76.207$ & \\
\hline Bilaterality & & & & 0.46 & & & 0.496 \\
\hline absent & 20 & $4(20.0)$ & 73.15 & & 1 & & \\
\hline present & 13 & $3(23.1)$ & 61.54 & & 1.753 & $0.348-8.823$ & \\
\hline ETE & & & & $\otimes 0.01$ & & & 0.945 \\
\hline No & 22 & $5(22.7)$ & 74.41 & & 1 & & \\
\hline Minimal/Gross & 11 & $2(18.2)$ & 50.00 & & 1.06 & $0.200-5.623$ & \\
\hline N stage & & & & 0.751 & & & 0.687 \\
\hline No & 10 & $3(30.0)$ & 60.95 & & 1 & & \\
\hline $\mathrm{N} 1 \mathrm{a}$ & 7 & $2(28.6)$ & 53.33 & & 0.99 & $0.130-7.597$ & \\
\hline $\mathrm{N} 1 \mathrm{~b}$ & 16 & $2(12.5)$ & 87.50 & & 0.49 & $0.090-2.685$ & \\
\hline Site of metastases & & & & 3.05 & & & 0.081 \\
\hline Pulmonary only & 21 & $2(9.5)$ & 80.00 & & 1 & & \\
\hline Extrapulmonary & 12 & $5(41.7)$ & 54.69 & & 3.87 & $0.846-17.723$ & \\
\hline Time of distant metastasis & & & & 1.13 & & & 0.288 \\
\hline Initial & 22 & $6(27.3)$ & 65.77 & & 1 & & \\
\hline Delayed & 11 & $1(9.1)$ & 80.00 & & 0.43 & $0.088-2.056$ & \\
\hline Symptom of distant metastases at initial diagnosis & & & & 12.44 & & & $\varangle 0.001$ \\
\hline Asymptomatic & 28 & $3(10.7)$ & 81.37 & & 1 & & \\
\hline Symptomatic & 5 & $4(80.0)$ & 20.00 & & 54.78 & $5.922-506.730$ & \\
\hline Adjuvant treatment modalities of distant metastases & & & & 14.18 & & & $\varangle 0.001$ \\
\hline RAl treatment & 24 & $1(4.2)$ & 90.91 & & 1 & & \\
\hline RAl combined with other treatment & 9 & $6(66.7)$ & 29.63 & & 27.69 & $4.917-155.993$ & \\
\hline${ }^{131}$ I avidity & & & & 1.87 & & & 0.171 \\
\hline No & 8 & $3(37.5)$ & 56.25 & & 1 & & \\
\hline Yes & 25 & $4(16.0)$ & 73.83 & & 0.28 & $0.047-1.724$ & \\
\hline RR-PTMC & & & & 7.60 & & & 0.006 \\
\hline No & 18 & $1(5.6)$ & 90.91 & & 1 & & \\
\hline Yes & 15 & $6(40.0)$ & 40.95 & & 8.94 & $1.884-42.458$ & \\
\hline Number of courses for ${ }^{131}$ I therapy & & & & 0.85 & & & 0.356 \\
\hline$\leq 3$ & 27 & $5(18.5)$ & 72.52 & & 1 & & \\
\hline$\varangle 3$ & 6 & 2(33.3) & 53.33 & & 2.61 & $0.341-19.929$ & \\
\hline & & Page 13 & & & & & \\
\hline
\end{tabular}




\begin{tabular}{|c|c|c|c|c|c|}
\hline Cumulative dose of ${ }^{131}$ I activities(GBq) & & & & 1.02 & 0.312 \\
\hline$\leq 22.2(600 \mathrm{mCi})$ & 28 & $5(17.9)$ & 72.73 & 1 & \\
\hline$₫ 22.2(600 \mathrm{mCi})$ & 5 & $2(40.0)$ & 53.33 & 2.92 & $0.365-23.371$ \\
\hline
\end{tabular}

Table 4. Prognostic factors of 10-year PFS rate for patients with PTMC and DM in multivariate analysis

\begin{tabular}{|c|c|c|c|c|}
\hline Variables & Ptients & HR & $95 \% \mathrm{Cl}$ & P-value \\
\hline Age (years) & & & & - \\
\hline$\varangle 55$ & 25 & - & & \\
\hline$\geq 55$ & 8 & - & - & \\
\hline Sex & & & & 0.005 \\
\hline Male & 9 & 1 & & \\
\hline Female & 24 & 0.02 & $0.001-0.294$ & \\
\hline Site of metastases & & & & - \\
\hline Pulmonary only & 21 & - & & \\
\hline Extrapulmonary & 12 & - & - & \\
\hline Symptom of distant metastases at initial diagnosis & & & & 0.022 \\
\hline Asymptomatic & 28 & 1 & & \\
\hline Symptomatic & 5 & 13.44 & $1.455-124.105$ & \\
\hline Adjuvant treatment modalities of distant metastases & & & & - \\
\hline RAl treatment & 24 & - & & \\
\hline RAl combined with other treatment & 9 & - & - & \\
\hline RR-PTMC & & & & 0.033 \\
\hline No & 18 & 1 & & \\
\hline Yes & 15 & 20.00 & $1.267-315.827$ & \\
\hline
\end{tabular}

Table 5. Clinical data for each PTMC patient with distant metastases 


\begin{tabular}{|c|c|c|c|c|c|c|c|c|c|c|c|c|c|}
\hline \multirow{2}{*}{$\begin{array}{l}\text { Patients } \\
\text { no. }\end{array}$} & \multirow[t]{2}{*}{ Sex } & \multirow{2}{*}{$\begin{array}{l}\text { Age } \\
\text { (years) }\end{array}$} & \multicolumn{3}{|c|}{ Primary tumor } & \multirow{2}{*}{$\begin{array}{l}\mathrm{N} \\
\text { stage }\end{array}$} & \multicolumn{3}{|l|}{ DM } & \multirow{2}{*}{$\begin{array}{l}\text { Time } \\
\text { (months) }\end{array}$} & \multicolumn{2}{|c|}{${ }^{131}$ I treatment } & \multirow{2}{*}{$\begin{array}{l}\text { Other } \\
\text { adjuvant } \\
\text { treatment } \\
\text { modalities }\end{array}$} \\
\hline & & & Histology & Bilateral & ETE & & Site & $\begin{array}{l}131 \text { I } \\
\text { avidity }\end{array}$ & $\begin{array}{l}\text { RR- } \\
\text { PTMC }\end{array}$ & & Courses & Doses(GBq) & \\
\hline 1 & $\mathrm{~F}$ & 34 & C-PTMC & $\mathrm{N}$ & $N$ & N1a & Lungs & Y & $N$ & 9 & 1 & 3.70 & No \\
\hline 2 & $\mathrm{~F}$ & 46 & C-PTMC & $\mathrm{N}$ & Gross & N1b & $\begin{array}{l}\text { Lungs } \square \\
\text { Brain! } \\
\text { Liver }\end{array}$ & Y & $\mathrm{N}$ & 1 & 4 & 25.90 & No \\
\hline 3 & $\mathrm{~F}$ & 47 & C-PTMC & $\mathrm{Y}$ & Minimal & No & Bones & $\mathrm{N}$ & $\mathrm{Y}$ & 0 & 1 & 5.40 & ER \\
\hline 4 & $\mathrm{~F}$ & 37 & C-PTMC & $\mathrm{Y}$ & $\mathrm{N}$ & N1b & Lungs & $\mathrm{Y}$ & $\mathrm{N}$ & 0 & 9 & 64.75 & No \\
\hline 5 & $\mathrm{~F}$ & 45 & C-PTMC & $\mathrm{N}$ & $\mathrm{N}$ & No & Bone & $\mathrm{Y}$ & $\mathrm{N}$ & 4 & 2 & 4.81 & No \\
\hline 6 & $\mathrm{~F}$ & 55 & C-PTMC & $\mathrm{N}$ & $\mathrm{N}$ & N1a & Lungs & $\mathrm{N}$ & $\mathrm{Y}$ & 7 & 1 & 3.70 & No \\
\hline 7 & $\mathrm{~F}$ & 37 & C-PTMC & $\mathrm{Y}$ & $N$ & N1b & Lungs & $\mathrm{Y}$ & $\mathrm{N}$ & 0 & 3 & 18.50 & No \\
\hline 8 & $\mathrm{~F}$ & 48 & FV-PTMC & $\mathrm{Y}$ & $N$ & No & Bones & Y & $\mathrm{Y}$ & 0 & 4 & 27.75 & PS,TKI \\
\hline 9 & $\mathrm{~F}$ & 35 & FV-PTMC & $\mathrm{N}$ & $N$ & N1b & Lungs & Y & $\mathrm{N}$ & 5 & 3 & 16.65 & No \\
\hline 10 & $\mathrm{~F}$ & 46 & C-PTMC & $\mathrm{N}$ & $N$ & No & Lungs & $\mathrm{N}$ & $\mathrm{Y}$ & 24 & 2 & 5.40 & No \\
\hline 11 & $M$ & 50 & C-PTMC & $N$ & $N$ & N1b & $\begin{array}{l}\text { Soft } \\
\text { tissue }\end{array}$ & Y & $N$ & 3 & 1 & 5.55 & No \\
\hline 12 & $\mathrm{~F}$ & 27 & C-PTMC & $\mathrm{N}$ & $N$ & N1b & Lungs & Y & $\mathrm{N}$ & 6 & 2 & 11.10 & No \\
\hline 13 & $\mathrm{~F}$ & 66 & C-PTMC & $\mathrm{N}$ & $N$ & $\mathrm{~N} 1 \mathrm{a}$ & Lungs & $\mathrm{N}$ & $\mathrm{Y}$ & 0 & 1 & 5.40 & TKI \\
\hline 14 & M & 60 & C-PTMC & $N$ & $N$ & NO & $\begin{array}{l}\text { Lungs } \square \\
\text { Bone }\end{array}$ & Y & Y & 0 & 6 & 44.40 & PS \\
\hline 15 & M & 62 & C-PTMC & $\mathrm{N}$ & $N$ & N1b & Bones & $\mathrm{N}$ & $\mathrm{Y}$ & 0 & 1 & 3.70 & PS \\
\hline 16 & M & 63 & C-PTMC & $\mathrm{Y}$ & Gross & N1a & Lung & Y & $\mathrm{N}$ & 0 & 2 & 12.95 & PS \\
\hline 17 & $\mathrm{~F}$ & 62 & C-PTMC & $N$ & $N$ & No & Bones & Y & $\mathrm{N}$ & 0 & 2 & 12.95 & ER \\
\hline 18 & $\mathrm{~F}$ & 53 & C-PTMC & $\mathrm{N}$ & $N$ & N1b & Lungs & Y & $\mathrm{N}$ & 0 & 1 & 5.40 & No \\
\hline 19 & $\mathrm{~F}$ & 69 & C-PTMC & $\mathrm{N}$ & Minimal & No & Bones & $\mathrm{Y}$ & $\mathrm{Y}$ & 0 & 3 & 20.35 & No \\
\hline 20 & $\mathrm{~F}$ & 28 & C-PTMC & $\mathrm{Y}$ & $N$ & N1a & Brain & Y & $N$ & 5 & 3 & 20.35 & ER \\
\hline 21 & $\mathrm{~F}$ & 21 & C-PTMC & $\mathrm{N}$ & $\mathrm{N}$ & $\mathrm{N} 1 \mathrm{~b}$ & Lungs & $\mathrm{Y}$ & $\mathrm{Y}$ & 1 & 1 & 5.55 & No \\
\hline 22 & $\mathrm{~F}$ & 42 & C-PTMC & $\mathrm{N}$ & Minimal & No & Lungs & Y & $\mathrm{Y}$ & 23 & 2 & 9.25 & No \\
\hline 23 & $\mathrm{~F}$ & 64 & C-PTMC & $N$ & $N$ & N1b & Lungs & Y & $N$ & 0 & 2 & 11.10 & No \\
\hline 24 & M & 33 & C-PTMC & $\mathrm{N}$ & $N$ & N1b & Lungs & Y & $N$ & 15 & 2 & 12.95 & No \\
\hline 25 & $\mathrm{~F}$ & 45 & C-PTMC & $\mathrm{N}$ & Minimal & N1b & Lungs & $\mathrm{N}$ & Y & 14 & 1 & 3.70 & No \\
\hline 26 & M & 34 & C-PTMC & $\mathrm{Y}$ & $N$ & N1b & Lungs & Y & $\mathrm{N}$ & 2 & 2 & 12.95 & No \\
\hline 27 & $\mathrm{~F}$ & 51 & C-PTMC & $\mathrm{Y}$ & Minimal & No & Lungs & Y & $\mathrm{N}$ & 1 & 5 & 29.60 & No \\
\hline 28 & $\mathrm{~F}$ & 41 & C-PTMC & $\mathrm{Y}$ & $N$ & N1a & Bones & Y & $N$ & $\otimes 12$ & 2 & 11.10 & No \\
\hline 29 & M & 33 & C-PTMC & $\mathrm{Y}$ & Minimal & N1b & Lungs & $N$ & $\mathrm{Y}$ & 11 & 2 & 5.40 & No \\
\hline 30 & $\mathrm{~F}$ & 40 & C-PTMC & $\mathrm{N}$ & Gross & $\mathrm{N} 1 \mathrm{a}$ & Lungs & $\mathrm{Y}$ & $\mathrm{Y}$ & 11 & 4 & 27.75 & No \\
\hline 31 & $\mathrm{~F}$ & 48 & C-PTMC & $\mathrm{Y}$ & Minimal & No & Lungs & $\mathrm{Y}$ & $\mathrm{N}$ & 0 & 3 & 16.65 & No \\
\hline 32 & M & 37 & C-PTMC & Y & Gross & N1b & Lungs & Y & Y & 3 & 2 & 18.50 & No \\
\hline 33 & M & 48 & C-PTMC & $\mathrm{Y}$ & $N$ & N1b & Bones & $\mathrm{N}$ & $\mathrm{Y}$ & 2 & 2 & 12.95 & $\mathrm{PS}, \mathrm{TKI}, \mathrm{CT}, \mathrm{E}$ \\
\hline
\end{tabular}

F: female, M: male; Age: age at diagnosis of distant metastases; Y: yes, N: no; Time: time between initial thyroidectomy and diagnosis of distant metastases; No:no other treatment modality; ER: external radiotherapy; PS: palliative surgery; TKI: tyrosine kinase inhibitor; CT: chemotherapy.

Figures 


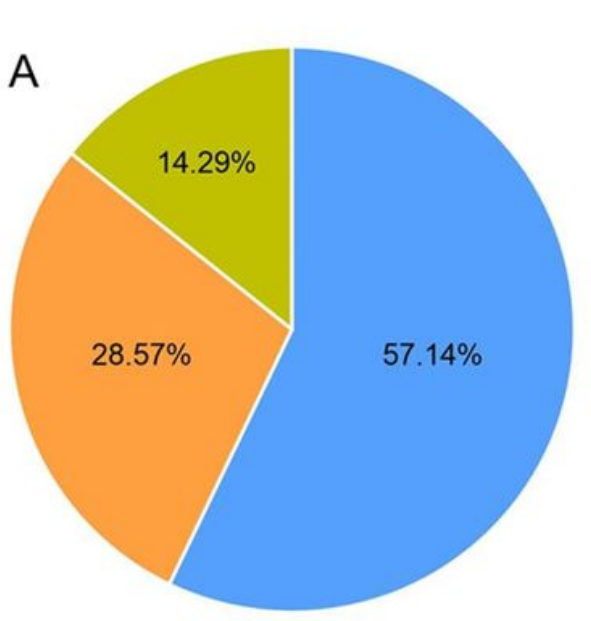

PD

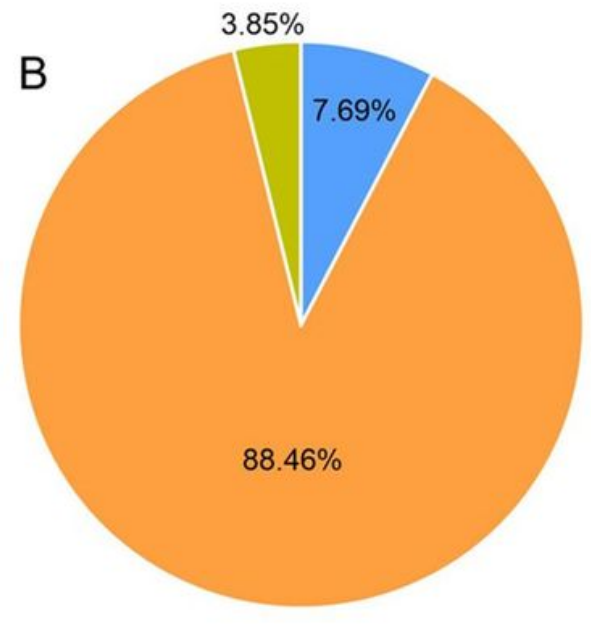

Non-PD

Figure 1

Changes in serum suppressed-Tg levels of PTMC patients with distant metastases in progression disease group (A) and non-progression group (B).
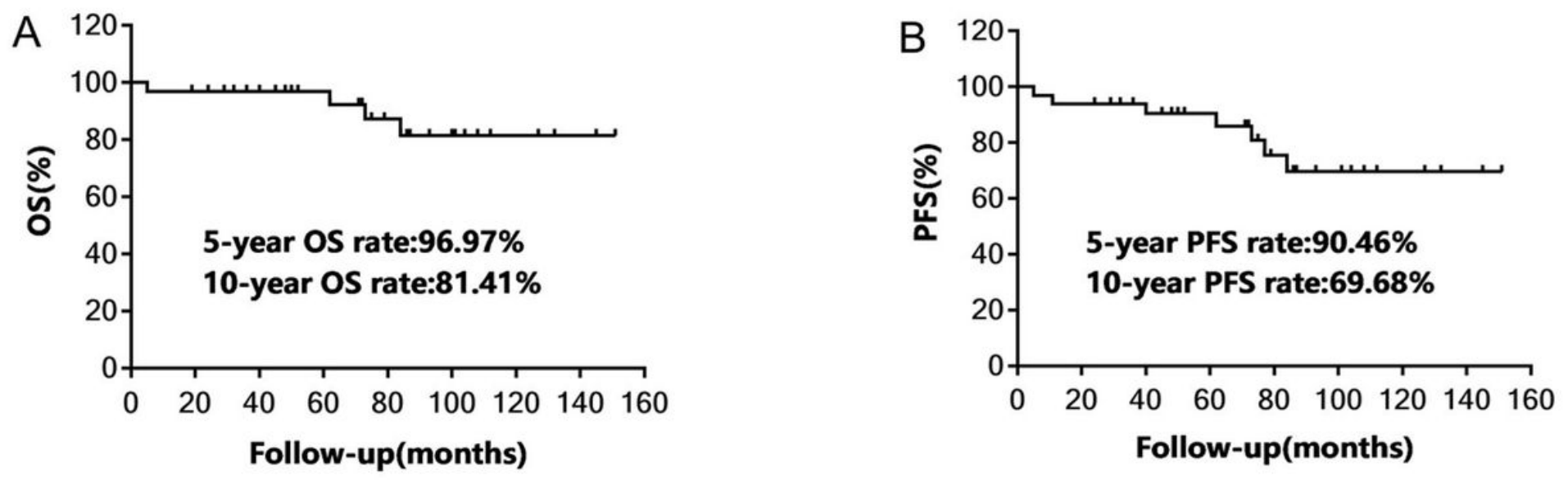

Figure 2

Kaplan-Meier curves for the overall survival (A) and progression-free survival (B) of PTMC patients with distant metastases. 

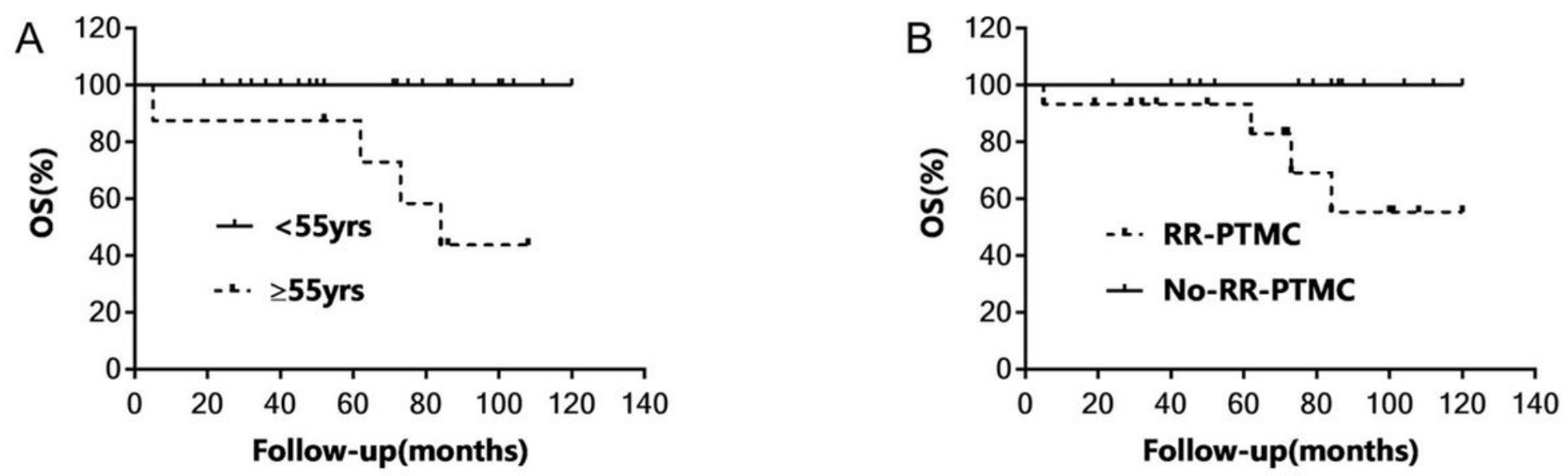

Figure 3

Univariate analysis of the prognostic factors for 10-year overall survival (OS) of all 33 PTMC patients with distant metastases; Comparison of 10-year OS curves for these PTMC patients with distant metastases according to the (A) age at diagnosis of distant metastases and (B) RR-PTMC status.
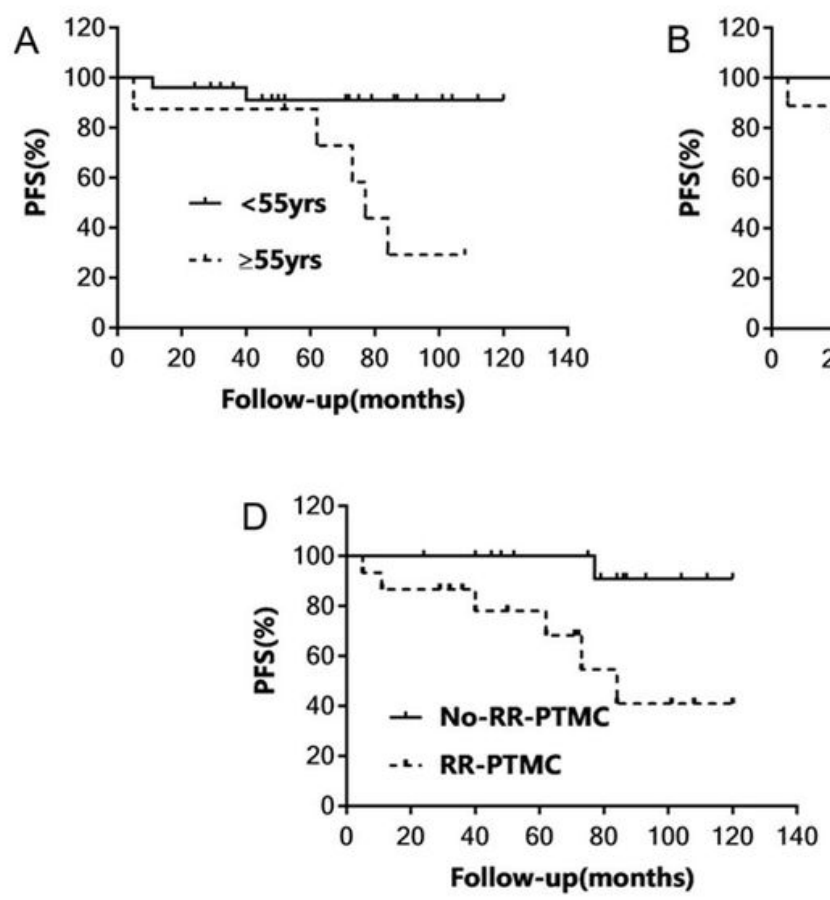
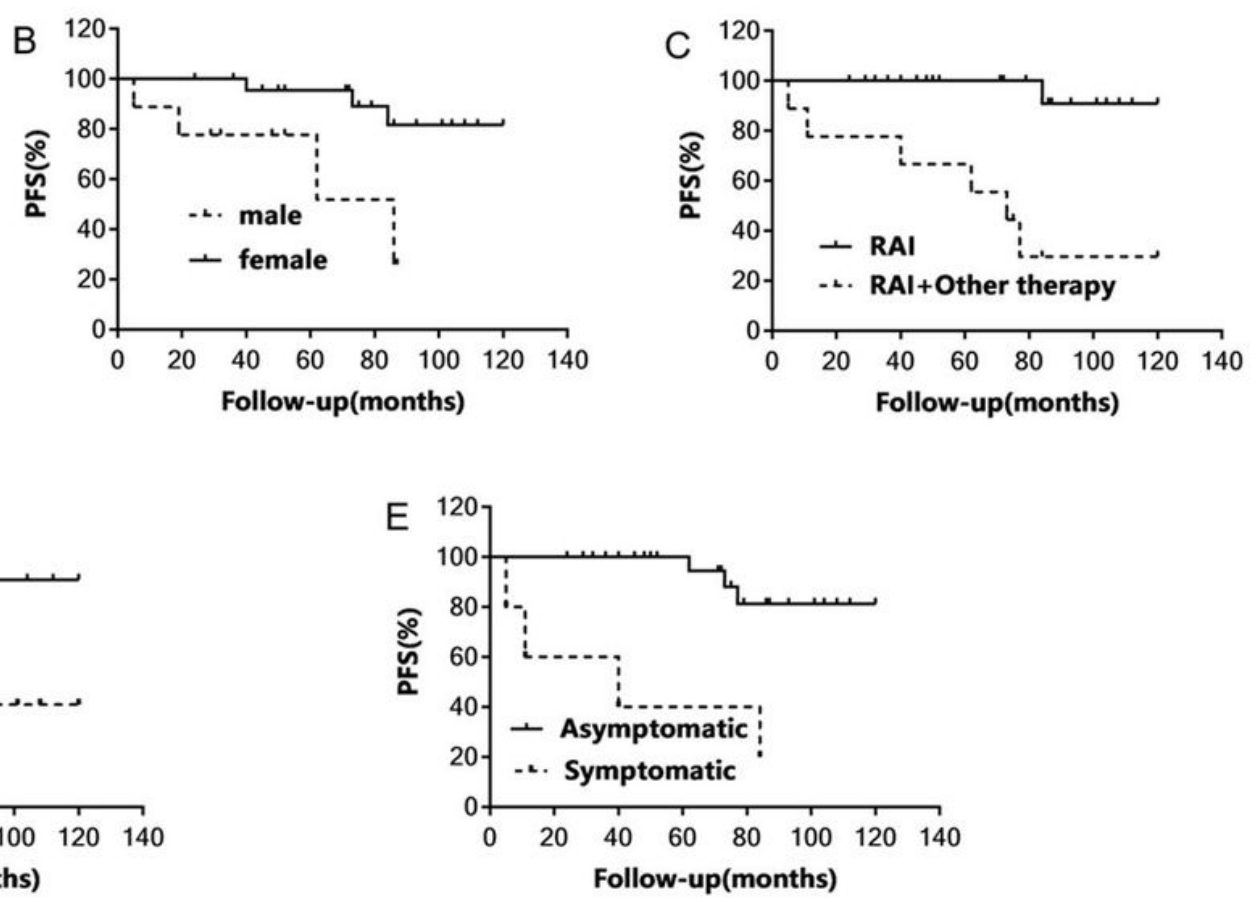

Figure 4

Univariate analysis of the prognostic factors for 10-year progression-free survival (PFS) of all 33 PTMC patients with distant metastases; Comparison of 10year PFS curves for these PTMC patients with distant metastases according to the (A) age at diagnosis of distant metastases, (B) gender,(C) adjuvant therapy modalities,(D) RR-PTMC status and (E) symptoms of distant metastatic disease. 